\title{
Task-Based Teaching and Layer Teaching Method Experiment in the Course Design of English Business Correspondence
}

\author{
Xianlan Zuo ${ }^{1, a,{ }^{*}, J u n h u a ~ Z h a n g ~}{ }^{2, b}$ \\ ${ }^{1}$ The School of Business, Ningbo City College of Vocational and Technology, Ningbo, China \\ ${ }^{2}$ The School of Information, Ningbo City College of Vocational and Technology, Ningbo, China \\ azuoxianlan_cn@163.com, bhangjunhua_cn@163.com \\ ${ }^{*}$ Corresponding author
}

\begin{abstract}
Thesis is divided into three parts. The first part introduces the task based-layer teaching five steps instructional design of English Business Correspondence in Business School of the Vocational Technology .The five-step design develops on the basis of traditional pre task, task and review, the tree-step teaching model, taking into account of the poor English of students in Vocational school, we add preparing lessons before class and exercising to consolidate after class the two rings .So the five-step is preview before class --- pre task: the situation creation, case introduction, leads to the task --- task: discuss a writing program, task decomposition, first attempt to writing step by step, later attempt to writing comprehensively -- after task: Showing, evaluation and learning model essay, self correction, after class: practicing, consolidating and improving. This design not only embodies the characteristics of task-based teaching, also shows the ideas and methods of hierarchical interactive teaching. In the second part of the paper, it gives the specific design of each step e of the task based- layer teaching in detail, taking the example of how to write the letter of amendment to the L/C, which clearly tells us how to carry out the whole process of five -step teaching. The third part introduces the writer's reflection of implementation of the task based- layer teaching five steps instructional design.
\end{abstract}

Keywords: task - based teaching method ; layer teaching ; English Business Correspondence ;L/C amendment

\section{高职任务驱动-分层互动型外贸英语函电教学活动设计 ——以撰写改证函为例}

\author{
左显兰 ${ }^{1, a^{*}}$, 张君华 ${ }^{2, b}$ \\ 1宁波城市职业技术学院商贸学院, 宁波, 中国 \\ 2宁波城市职业技术学院信息学院, 宁波, 中国 \\ azuoxianlan_cn@163.com, bhangjunhua_cn@163.com \\ *通讯作者
}

中文摘要：论文分三部分，第一部分介绍了任 务驱动--分层教学高职外贸英语五阶段教学阶段设 计, 该设计是在传统任务前、任务中、任务后三阶 段教学模式的基础上, 考虑到高职经贸类学生英语 基础比较薄弱这一现实, 在这三阶段模式前后分别 加了课前预习, 课后练习巩固这两环。即课前预
习------任务前:创设情境、导入案例、引出任务----任务中: 讨论写作方案、分解任务、先分步后综合 尝试写作------任务后: 展示评价、学习范文、自我 矫正------课后练习、巩固提高, 五阶段。论文第二 部分, 以攥写改正函为例, 详细介绍了任务驱型外 贸英语函电分层教学各阶段的具体设计。论文第三 
部分介绍了笔者任务驱动-分层互动型外贸英语函 电教学活动设计方案实施后的反思。

关键词: 任务驱动型教学 分层教学 外贸 函电信用证修改

\section{1. 引言}

外贸英语函电是高职外贸英语专业和 国际经贸专业高年级学生开设的一门必修 课。笔者在高职商务学院从事外贸函电教学 多年, 始终觉得经贸类学生对这门课程的学 习效果不甚理想, 高职 “项目导向, 任务驱 动型” 教学改革似乎给笔者指明了教改的方 向。笔者在讲授 “L/C AMENDMENT” 这 一环节时, 对任务驱动型教学进行了有意的 尝试, 收到了比以往要好的教学效果, 曾公 开发表了《任务驱动型高职经贸类外贸英语 函电教学的有意尝试》一文。此后笔者进一 步将分层教学、支架式教学等方法引入任务 驱动型课程设计中, 教学效果更好, 为此笔 者再一次以撰写信用证修改函为例, 具体介 绍任务驱动-分层教学法在高职型外贸英语 函电教学设计方案、实施步骤及心得体会。

\section{2. 任务驱动--分层教学高职外贸英语教学 阶段设计}

“任务驱动法”是一种建构主义的学习 理论指导下的教学方法, 是基于探究学习和 协作学习的学习模式。其显著特征是: 以发 展学生的能力为主, 把教师的功能定位在 “促进学生学习, 引导学生成功”上。英国 著名学者Andrew Little john 作了以下 解释: 任务型教学活动是一种完成任务的活 动, 学生在活动中真实的使用语言。任务型 教学法是 20 世纪 80 年代外语教学研究和第 二语言习得研究提出的重要的语言教学方 法和教学模式。其核心内容是教学过程以任 务为主线, 教师为主导, 学生为主体, 习得 语言。

任务型教学法的教学阶段, 不同的学者 提出了大致相同的三阶段的的教学模式: 即 任务前阶段、任务中阶段和任务后阶段。

“分层互动” 的教学模式, 实际上是一 种课堂教学的策略。这里的 “分层” 有两层 含义, 一是将教学内容由简到易分层, 二 是将学生进行良莠搭配的一种隐性的分层。
笔者在教授 “L/C AMENDMENT”这 一项目时, 对任务驱动型教学在不同学期进 行了多次有意的尝试, 也基本沿用了上述三 阶段的教学模式。只是考虑到高职学生英语 基础比较薄弱这一现实, 在这三阶段模式前 后分别加了课前预习, 课后练习巩固这两 环。五阶段的教学设计在不同实验阶段, 实 施实施过程细节上有些变化, 如下图

\begin{tabular}{|c|c|c|c|c|}
\hline 课前预习 & $\begin{array}{l}\text { 任务前: 创设 } \\
\text { 情景, 导入案 } \\
\text { 例, 引出任务 }\end{array}$ & $\begin{array}{l}\text { 任务中: 讨论写作方 } \\
\text { 案、分解任务, 先分 } \\
\text { 步后综合尝试写作 }\end{array}$ & $\begin{array}{l}\text { 任务后: 展示 } \\
\text { 评价, 学习范 } \\
\text { 文, 自我矫正 }\end{array}$ & $\begin{array}{l}\text { 课后练 } \\
\text { 习, 巩 } \\
\text { 固提高 }\end{array}$ \\
\hline
\end{tabular}

图1:任务驱动-分层互动型教学模式阶段设计

这五阶段的教学设计和前文《任务驱动 型高职经贸类外贸英语函电教学的有意尝 试》中提到的五阶段基本一致, 前文五阶段 的教学设计如

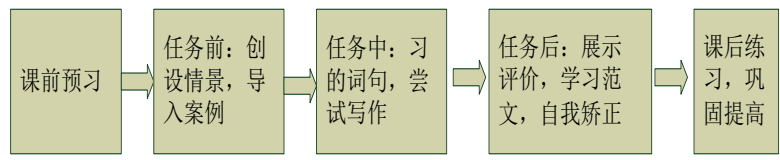

图2: 任务驱动型教学模式阶段设计

从图一与图二的比较可以看出, 任务驱 动-分层互动型教学模式和以前单纯任务驱 动型教学模式相比, 五个阶段是一致的, 所 不同的是, 在执行任务的过程中, 具体设计 方案变化较大, 多了 “讨论写作方案, 任务 分解和先分步后综合” 字样, 看似几个字的 差别, 但教学活动设计的理念和活动实施的 方案都发生了很大的变化。“讨论写作方案” 体现了师生, 和生生之间分组合作互动的过 程。

“任务分解, 先分步后综合” 体现了 将任务按照难易程度由简到易的分层思想。 老师把任务分解, 引导学生一部分一部分, 甚至是一句话一句话的去写, 在写作过程 中，时时给予 “支架支持”（即专业词汇和 句式）, 这样降低了学生的学习难度, 消 除了学生的畏惧心理, 学生学习的积极主动 性会更高, 而且分步实施写作, 学生词句的 学习和明确的任务紧密相连, 学生学得更认 真, 记得也更牢固。

\section{3. 任务驱动型外贸英语函电教学各阶段的 具体设计}

改证函撰写计划用3课时, 下面对这一 过程各阶段的教学设计进行详细描述。 
3.1 课前预习: 预习内容简介, 词汇和句子

外贸英语函电每一章节前, 都有一个 brief introduction, 课前我们布置学生预习, 既可以使他们巩固外贸实务知识, 又可以提 高他们的英语水平, 为他们课堂上顺利完成 任务打下基础。

\section{2 任务前: 创设情景, 导入案例 (5分钟)}

在创设情景, 导入案例这一环节的教 学, 可以采用交际法进行, 教师用英语设计 一个个问题, 引导学生讨论、交流和回答。 比如在针对信用证的修改, 笔者设计了以下 几个问题:

Question 1.On receiving the $\mathrm{L} / \mathrm{C}$, what' $\mathrm{s}$ the first thing that the seller should do?

Stutents Answer: (略)

Queation2.if the seller find some discrepancies (不符点)between the L/C and the S/C, what should they do? Why?

Stutents Answer: (略)

鉴于高职经贸类学生英语基础相对薄 弱这一现状，老师在用英语提问的时候，一 定要用PPT 呈现问题, 有些难一点的单词 后面要加注汉语, 确保学生能听懂问题。问 题提出后, 可以允许学生分组讨论交流, 然 后回答。在充分调动学生已有知识经验的基 础上, 老师再导入案例, 布置任务。

表1 案例中信用证与合同不符之处

\begin{tabular}{|r|c|c|}
\hline 不符点 & 信用证规定 & 合同规定 \\
\hline 1 & 不允许转船 & 允许转船 \\
\hline 2 & $\begin{array}{c}\text { 8-9月份分两个 } \\
\text { 月平均装运 }\end{array}$ & $\begin{array}{c}\text { 9月30号之前 } \\
\text { 一批装运完 }\end{array}$ \\
\hline 3 & $\begin{array}{c}\text { 数量和金额前 } \\
\text { 没有” 大约” 字样 }\end{array}$ & $\begin{array}{c}\text { 数量和金额前 } \\
\text { 有” 大约”字样 }\end{array}$ \\
\hline
\end{tabular}

Today we will study how to write the letter asking for amending the $\mathrm{L} / \mathrm{C}$. The following is a case : 宁波埃美柯公司收到美 国威高公司向其开立的信用证后, 通过单证 员仔细审核, 发现有下列三条条款与两家公 司先前达成的销售合同不符。假设你作为埃 美柯公司业务员, 为此, 致函威高公司要求 修改信用证中的不符之处。
3.3 任务中: 讨论写作方案、任务分解, 先 分步, 后综合尝试写作 (75分钟)

第一步: 讨论写做方案和步骤 (10分钟)

在导入案例, 引出任务之后, 接下来就 是引导学生结合案例和任务, 分组讨论写作 方案和步骤, 然后分组汇报讨论结果。学生 汇报了两种方案的写作步骤, 陈列如下:

\section{Formal one :}

Step 1.Expressing your thanks for the incoming $\mathrm{L} / \mathrm{C}$ and stating your letter-replying purpose的)

Step 2.Indicaing the discrepancies

Step 3.Requesting the applicant to make the necessary amendments

Step 4.Expressing your expectation

\section{Formal two:}

与方案一不同的是, 第二步就直接给出 修改建议 (Giving your proposals on 1/c amendment）其它步骤和第一个方案一样

第二步: 根据上述给出的写作方案和步 骤, 结合案例, 分步尝试写作 (50分钟)

下面我们以方案一的写作步骤为例, 进 行具体阐述。

阶段任务一: 对来证表示感谢, 常用的 两句话翻译

1.收到你方编号为NH18的信用证，非 常感谢。2.但通过仔细审核, 我们发现有下 列不符点。

教师用PPT呈现汉语, 要求学生以小组 为单位逐句翻译, 然后分组汇报翻译结果。 这两句话中涉及到的关键词句 “不符点

(discrepancies)）。“仔细审核（make a thorough examination或 on perusal )”，教 师可以给出, 帮助学生搭建翻译支架。学生 翻译完毕, 教师可用PPT呈现尽可能多的正 确结果。

\section{阶段任务二: 指出不符点}

指出信用证与合同的不符点, 是这封改 证函最核心的内容, 难度也是最高的。笔者 没有要求所有学生一口气就完成三个不符 点的笔译, 而是把任务进一步分解, 一个个 不符点逐一笔译。并且在笔译每个不符点 时, 并不是一开始就写整句话, 而是从关键 词汇和短句翻译开始，扫除写作中的障碍 后, 再让学生独立写出整句话, 由易到难, 循序提高。具体如下: 
点。

\section{首先, 要求学生用英语写出第一个不符}

\begin{tabular}{|r|c|c|}
\hline 不符点 & 信用证规定 & 合同规定 \\
\hline 1 & 不允许转船 & 允许转船 \\
\hline
\end{tabular}

在写整句话之前, 老师先引导学生口头 翻译几个专业词汇和短句 “转船”、“允许 转船”和“规定”的英语表述, 并提醒用“but” 转折句型来写。这样学生写作的难度就大大 降低了。学生写完后, 分小组汇报结果, 老 师点评并用PPT呈现正确的结果。

然后用同样的方法, 引导学生写出第二

个，第三个不符点。

阶段任务三:要求开证申请人对信用证 做出必要的修改, 学生翻译下面两句话

1. 请立即做出必要的修改 2. 否则我们 不能按期装船，教学方法同阶段任务一。

阶段任务四: 表达作者的期望, 要求学 生翻译下面一句话

1.我们盼望早日收到你方的改证。

这句话比较简单, 学生口头翻译就可。

第三步, 要求学生根据上述案例, 独立 撰写一封完整的改证函, 信函上传到天空教 室 (15分钟)

这时学生进入网络课程, 在作业任务栏 中打开老师布置的任务, 在电脑上写一封完 整的改证函。同学们在写作的过程中, 老师 可以在教室巡回走动, 对需要帮助的学生进 行个别辅导, 也可以用教师主机集域电子教 室监控平台查看学生的写作情况, 在主机上 对学生进行遥控辅导。

\section{4 任务后: 展示作品, 学习范文, 矫正 答案 (40分钟)}

在学生独立完成信函写作后, 我们可以 用机房集域电子教室把部分学生的作品广 播, 展示给全班同学, 一起来审阅, 这样全 班同学都是评判员, 也是被评的对象, 可以 让他们真正参与到学习活动中来。除了全班 投影部分学生的作品外, 也可以小组同学们 相互交换评阅, 分小组推荐优秀的作品展 出。

在展示完学生的作品后, 我们可以把这 封信的范文用PPT呈现给全班同学, 一起来 学习样信。这和传统的教学步骤正好颠倒过 来了。这样学生很快就可以发现自己和好的 范文之间的差距。同时, 学生在尝试写作的 过程中, 难免会遇到各种问题, 学生带着问
题来学习样信, 学习会更加积极主动, 学习 效果会更好。

\section{5 课后巩固提高}

课后学生要做些词汇、短语和句子的英 汉互译, 并用两种方案, 完成上述案例的情 景写作练习。作业布置和评阅的方式也可以 多样化，除了传统的纸和笔的方式完成作业 外, 还可以尝试一些现代化的沟通和交流方 式, 比如教这门课程的教师可以共同建立一 个外贸英语函电的课程网站, 建立习题库, 案例集, 在网上布置作业, 学生网上和老师 交流。没有建立课程网站的教师可以开通自 己的BLOG, 或建立QQ群, 群里和学生讨 论一些共同的问题。

\section{4. 任务驱动-分层互动型外贸英语函电教 学活动设计方案实施的反思}

以上是任务驱动-分层互动型外贸英语 函电教学活动设计方案及实施步骤, 整个教 学过程以任务为主线来展开, 任务由易到 难, 分层实施, 层层递进, 学生按学习能力 分层分组, 学生一环紧扣一环, 始终是学习 活动的主体, 教师只是指导者和引路人, 适 时给予支架支持。这样能很好地调动学生学 习的积极主动性, 使其学得更好。这为学生 走上工作岗位独立缮写外贸信函做好了充 分的准备。

当然在实施的过程中, 笔者有些心得或 困惑, 有必要提出来和大家讨论。

\section{1 任务的设计要紧密结合地方外贸实 际, 具有真实性}

上述这个任务要求学生以宁波埃美柯 公司的名义给美国高乐公司写信。因为同学 们身处宁波, 都知道埃美柯公司生产的水龙 头很有名, 这样就有一种亲近感和真实感。

\section{2 任务要合理分解, 分层设计, 分步实 施, 具有可操作性并兼顾学生的个体差异}

笔者在要学生完成一封改证函时, 考虑 到高职学生英语水平和能力的差异, 先是引 导学生讨论写作方案和步骤, 然后根据写作 方案和步骤, 结合案例一步一步来写。甚至 在最核心的第二步，指出信用证与合同的不 符点时, 是一个个不符点都分开来完成, 每 
个不符点都是先翻译核心词汇, 短语, 再翻 译整个句子。

\section{3 学生要按能力差异, 分层分组, 整个 活动老师要精心设计, 善于组织}

教师和学生在任务中的角色要准确定 位。老师是活动的组织者和指导者, 是学生” 学习支架” 的搭建者, 学生是任务的执行者 和活动的主体。教师要发挥自己的主导作 用, 让学生都参与到学习活动中来, 就需要 照顾到个体差异, 将学生按照学习能力进行 良莠搭配, 分层分组, 让学生之间能互相给 予支架支持, 充分发挥学生的智慧, 构建合 作学习小组。本例中学生合作就给出了两种 不同的写作方案。

\section{4 建立合理的激励机制, 调动学生学习 的积极主动性, 是任务顺利完成的关键}

学生积极主动的课前预习, 是上课时老 师创设情景, 导入案例的关键, 否则, 教师 提问, 没人回答, 开局不利, 势必影响后面 的环节。所以笔者学期开始, 就给同学们讲 明本门课的考核标准主要是形成性教学评 价为主, 平时学生的课堂回答问题, 任务参 与和完成情况占 $70 \%$, 期末考试占 $30 \%$ 。课 堂上学生每主动答对一个问题, 就可以得 1 分, 这样以来, 笔者每提一个问题, 或布置 一个翻译任务, 学生都争先恐后抢答, 气氛 非常活跃。

\section{5 学生任务完成后, 整体结果如何评价 是待进一步讨论的问题}

在任务分解, 分步实施时, 学生的汇报 可以采用抢答式, 并且任务简单, 汇报比较 容易, 老师比较好评价。在分步实施后, 要 学生完成整封信, 信函上传到课程网站后, 教师在课堂上集中展示和点评的作品的份 数毕竟有限, 虽然有分组, 如何发挥小组评 价的功能, 发挥各小组长在学生整体结果评 价中的功能还需要进一步探讨。

\section{5. 结束语}

以上以撰写改证函为例, 详细介绍了任 务驱动-分层教学外贸英语函电教学设计方 案, 实施步骤和实施后的一些心得体会, 希 望能起到抛砖引玉的作用。

\section{致谢}

本文为浙江省2013年课堂教学改革项目《高职 任务驱动-导生制合作学习课堂教学模式研究课题》 （课题编号 kg2013882）的阶段成果之一, 主持人: 左显兰; 浙江省高职高专专业带头人专业领军项目 （编号1j2013174）、浙江省教育厅科研项目（编号 Y201430684）研究成果之一, 项目主持人张君华。

\section{References}

[1]. Zheng Dehua. Discusing on task-based teaching of Business English correspondence by $\lceil\mathrm{J}\rceil$. Neijiang Economy, vol.2, pp. 108, 2008

[2]. Wang Liyang. Research on the reform of task-based English teaching [J]. New Western. vol.20, pp. 171, 2007

[3]. Feng Wei. Task-based foreign language classroom teaching activity design [J]. Technology Information. vol.6, pp. 146, 2007

[4]. zuo-Xianlan. Have a try of task-based teaching in the course of English business correspondence in the VET college $[\mathrm{J}]$. Consumer Guide, vol8, pp. 172-173, 2008

[5]. Zhang Junhua, Zuo Xianlan. Task -based-tutors system and cooperative learning: an effective mode of improving the classroom activities in Higher Vocational Colleges $[\mathrm{J}\rceil$. Vocational Education Communications, vol18, pp. 94-99, 2012

[6]. He yue. The business English teaching based on the scaffolding teaching mode. Journal of Wuxi Institute of Technology[J]. vol3, pp. 78-90., 2012

[7]. Chen Huizhen. Research on the existing problems and countermeasures in foreign trade English correspondence teaching of VET College「J]. Jiangsu Institute of Education Journal (SOCIAL SCIENCE EDITION), vol.3, pp. 113-114, 2008 